\title{
Whipple disease mimicking inflammatory bowel disease
}

\author{
Maiko Tatsuki ${ }^{1}$, Takashi Ishige ${ }^{1}$, Yoshiko Igarashi ${ }^{1}$, Reiko Hatori $^{1}$, Akira Hokama $^{2}$, Junko Hirato ${ }^{3}$, Aleixo Muise ${ }^{4}$, \\ Takumi Takizawa ${ }^{1}$, Hirokazu Arakawa ${ }^{1}$ \\ ${ }^{1}$ Department of Pediatrics, Gunma University Graduate School of Medicine, Maebashi; ${ }^{2}$ Department of Endoscopy, University of the Ryukyus, \\ Nishihara, ${ }^{3}$ Clinical Department of Pathology, Gunma University Hospital, Maebashi, Japan; ${ }^{4}$ Cell Biology Program, Inflammatory Bowel \\ Disease Centre, The Hospital for Sick Children, Toronto, ON, Canada
}

Whipple disease is a systemic chronic infection caused by Tropheryma whipplei. Although chronic diarrhea is a common gastrointestinal symptom, diagnosis is often difficult because there are no specific endoscopic findings, and the pathogen is not detectable by stool culture. We present a female patient with Whipple disease who developed chronic bloody diarrhea and growth retardation at the age of 4 years. Colonoscopy showed a mildly edematous terminal ileum and marked erythema without vascular patterns throughout the sigmoid colon and rectum. Subsequently, a primary diagnosis of ulcerative colitis was made. Histopathological analysis of the terminal ileum showed the presence of foamy macrophages filled with periodic acidSchiff-positive particles. Polymerase chain reaction using DNA from a terminal ileum biopsy sample amplified a fragment of 16S rRNA from T. whipplei. Antibiotic treatment relieved the patient's symptoms. There was no evidence of immunodeficiency in the present case. Since Whipple disease worsens after anti-tumor necrosis factor inhibitor therapy, considering this infection in the differential diagnosis may be important in patients with inflammatory bowel disease, especially before initiation of immunotherapy. (Intest Res 2021;19:119-125)

Key Words: Tropheryma whipplei; Colitis, ulcerative; Child, preschool

\section{INTRODUCTION}

The incidence of pediatric onset inflammatory bowel disease (PIBD) is increasing worldwide. ${ }^{1}$ Its diagnosis is based on a combination of history, physical, and laboratory examinations, as well as gastrointestinal (GI) endoscopy with histology. ${ }^{2}$ Immunomodulators are often used for the treatment of PIBD. However, these agents can worsen clinical symptoms of patients with infectious diseases; therefore, excluding enteric infections before deciding the course of therapy is crucial. ${ }^{2}$ In addition to bacterial infections (e.g., infection with Salmonella, Shigella, Yersinia, Campylobacter, and Clostridium difficile),

Received December 30, 2019. Revised March 21, 2020.

Accepted April 15, 2020.

Correspondence to Takashi Ishige, Department of Pediatrics, Gunma

University Graduate School of Medicine, 3-39-22, Showa-machi, Maebashi

371-8511, Japan. Tel: +81-272208205, Fax: +81-272208215, E-mail:

ishiget@gunma-u.ac.jp viral infections (e.g., infection with cytomegalovirus and Epstein-Barr virus) should also be excluded. ${ }^{2}$ Some rare infectious agents may result in chronic intestinal inflammation resembling PIBD.

The bacteria, Tropheryma whipplei, is the causative agent of the rare chronic infectious disease, Whipple disease (WD). ${ }^{3}$ Several cases with GI symptoms have been reported in adults, ${ }^{4,5}$ but no case has been reported in non-immunocompromised children. Moreover, cases with prolonged bloody diarrhea and chronic inflammation of the colon, resembling IBD, have never been reported. Here, we report a case of WD diagnosed initially as PIBD that was successfully treated with antibiotics. All genetic experiments were carried out with the approval of the Research Ethics Board at Gunma University Hospital, Maebashi, Japan, and the Hospital for Sick Children, Toronto, Canada. Informed consent to participate in research was obtained from all participants. 


\section{CASE REPORT}

A 4-year-old female developed diarrhea and hematochezia. She was born via a caesarean section without any complications. She did not have a history of symptoms suggestive of immune dysfunction, such as prolonged fever or opportunistic infections. Her mother had Crohn's disease (CD) since the age of 10 years and was refractory to infliximab. None of her other relatives had any symptoms of immunodeficiency or inflammatory bowel disease (IBD). At the age of 4 years and 1 month, the patient's bowel movement increased to four times per day and was accompanied by bloody stool. She was admitted at a regional hospital and underwent colonoscopy, which showed chronic colitis. Considering her family history, she was diagnosed with ulcerative colitis, and treated with oral mesalazine and exclusive enteral nutrition using elemental formula. Although her diarrhea symptoms improved, her symptoms relapsed soon after she resumed taking food. She was subsequently referred to our hospital at the age of 4 years and 9 months for further treatment.

At the time of admission, she showed growth retardation with poor weight gain; her growth velocity was 2 standard deviations below average. On physical examination, she was afebrile and had stable vital signs and her laboratory data is presented in Table 1. No infectious agents were identified in the collected stool specimens; Clostridium difficile toxin was not detected in a stool sample. The duodenal mucosa appeared slightly erythematous and edematous on esophagogastroduo-

Table 1. Laboratory Data at the Time of Inflammatory Bowel Disease Diagnosis

\begin{tabular}{|c|c|c|c|}
\hline Variable & Value & Variable & Value \\
\hline Hematology & & $\mathrm{Na}(\mathrm{mEq} / \mathrm{L})$ & 142 \\
\hline Leukocytes $\left(\times 10^{3} / \mu \mathrm{L}\right)$ & 7.4 & $\mathrm{~K}(\mathrm{mEq} / \mathrm{L})$ & 4.1 \\
\hline Red blood cells $\left(\times 10^{3} / \mu \mathrm{L}\right)$ & 4,300 & $\mathrm{Cl}(\mathrm{mEq} / \mathrm{L})$ & 109 \\
\hline Hemoglobin (dL) & 11.2 & Total bilirubin (mg/dL) & 0.2 \\
\hline Platelets $\left(\times 10^{3} / \mu \mathrm{L}\right)$ & 388 & AST (IU/L) & 25 \\
\hline ESR $(\mathrm{mm} / \mathrm{hr})$ & 29 & ALT (IU/L) & 8 \\
\hline Serology & & $\mathrm{LDH}(\mathrm{IU} / \mathrm{L})$ & 224 \\
\hline $\mathrm{CRP}(\mathrm{mg} / \mathrm{dL})$ & 0.08 & $\gamma-\mathrm{GTP}(\mathrm{IU} / \mathrm{L})$ & 12 \\
\hline $\lg G(\mathrm{mg} / \mathrm{dL})$ & 803 & CPK (IU/L) & 64 \\
\hline $\lg A(m g / d L)$ & 176 & Amylase (IU/L) & 70 \\
\hline $\lg \mathrm{M}(\mathrm{mg} / \mathrm{dL})$ & 135 & $\mathrm{Fe}(\mu \mathrm{g} / \mathrm{dL})$ & 19 \\
\hline Urinalysis & & UIBC ( $\mu \mathrm{g} / \mathrm{dL})$ & 292 \\
\hline Glucose & - & Antinuclear antibody & 40 \\
\hline Protein & - & PR-3 ANCA & $<10$ \\
\hline Occult blood & - & MPO-ANCA & $<10$ \\
\hline Stool analysis & & Cytomegalovirus PCR & - \\
\hline Occult blood & + & EBV-lgM & - \\
\hline Culture & Normal flora & EBV-lgG & $<25$ \\
\hline Serum biochemistry & & $\beta$-D-glucan & $<3.3$ \\
\hline Total protein $(\mathrm{g} / \mathrm{dL})$ & 6.6 & Candida antigen & - \\
\hline Albumin (g/dL) & 3.9 & Interferon- $\gamma$ release assays & - \\
\hline Glucose (mg/dL) & 106 & $\mathrm{HBsAg}$ & - \\
\hline Urea nitrogen (mg/dL) & 15 & Anti-HCV & - \\
\hline Creatinine (mg/dL) & 0.23 & Anti-HIV & - \\
\hline
\end{tabular}

ESR, erythrocyte sedimentation rate; CRP, C-reactive protein; Ig, immunoglobulin; AST, aspartate aminotransferase; ALT, alanine aminotransferase; LDH, lactic dehydrogenase; $\gamma$-GTP, gamma-glutamyl transpeptidase; CPK, creatine phosphokinase; UIBC, unsaturated iron binding capacity; PR-3 ANCA, proteinase 3 anti-neutrophil cytoplasmic antibody; MPO-ANCA, myeloperoxidase antineutrophil cytoplasmic antibody; PCR, polymerase chain reaction; EBV, Epstein-Barr virus; HBsAG, hepatitis B surface antigen; HCV, hepatitis C virus; HIV, human immunodeficiency virus. 

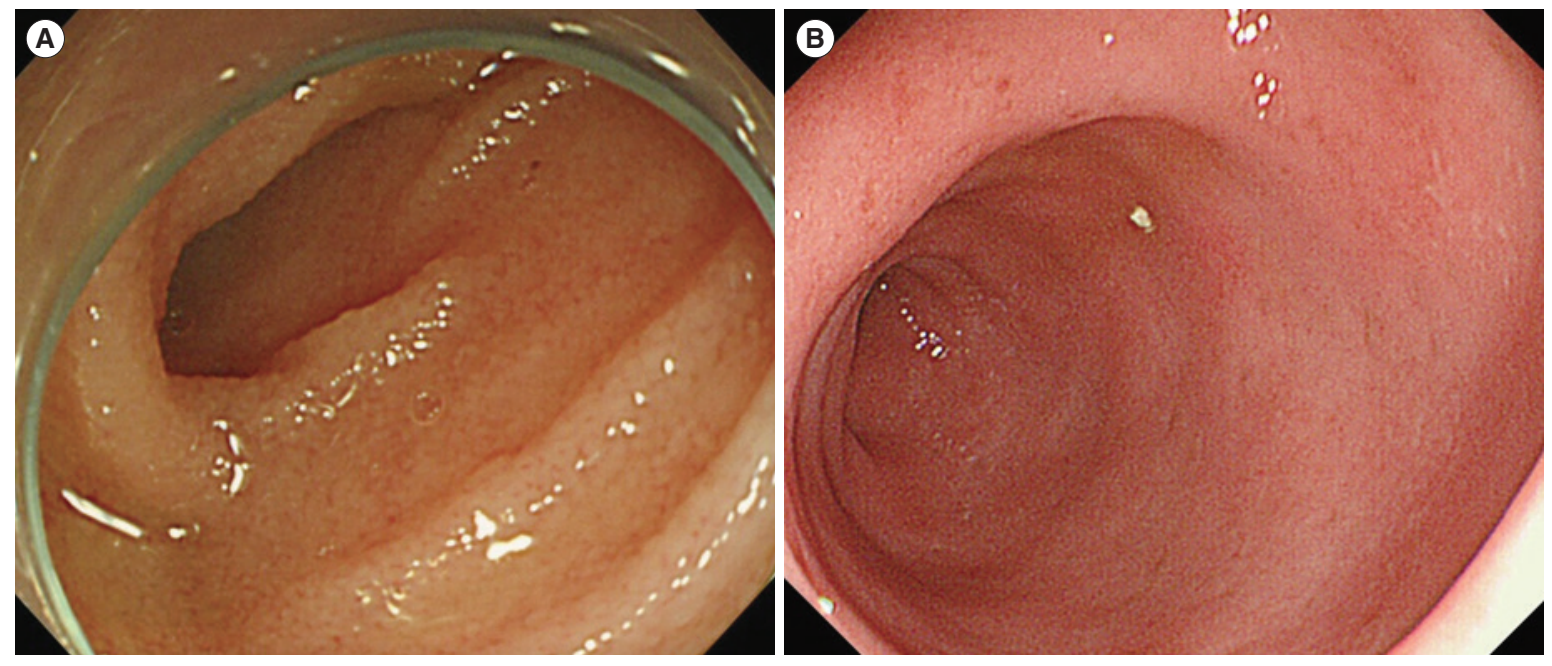

Fig. 1. Endoscopic image of the terminal ileum (A) and sigmoid colon (B) of the patient at the time of admission. The ileum showed mild edema. Colonoscopy showed friability, erythema, absence of vascular patterns, and erosions.
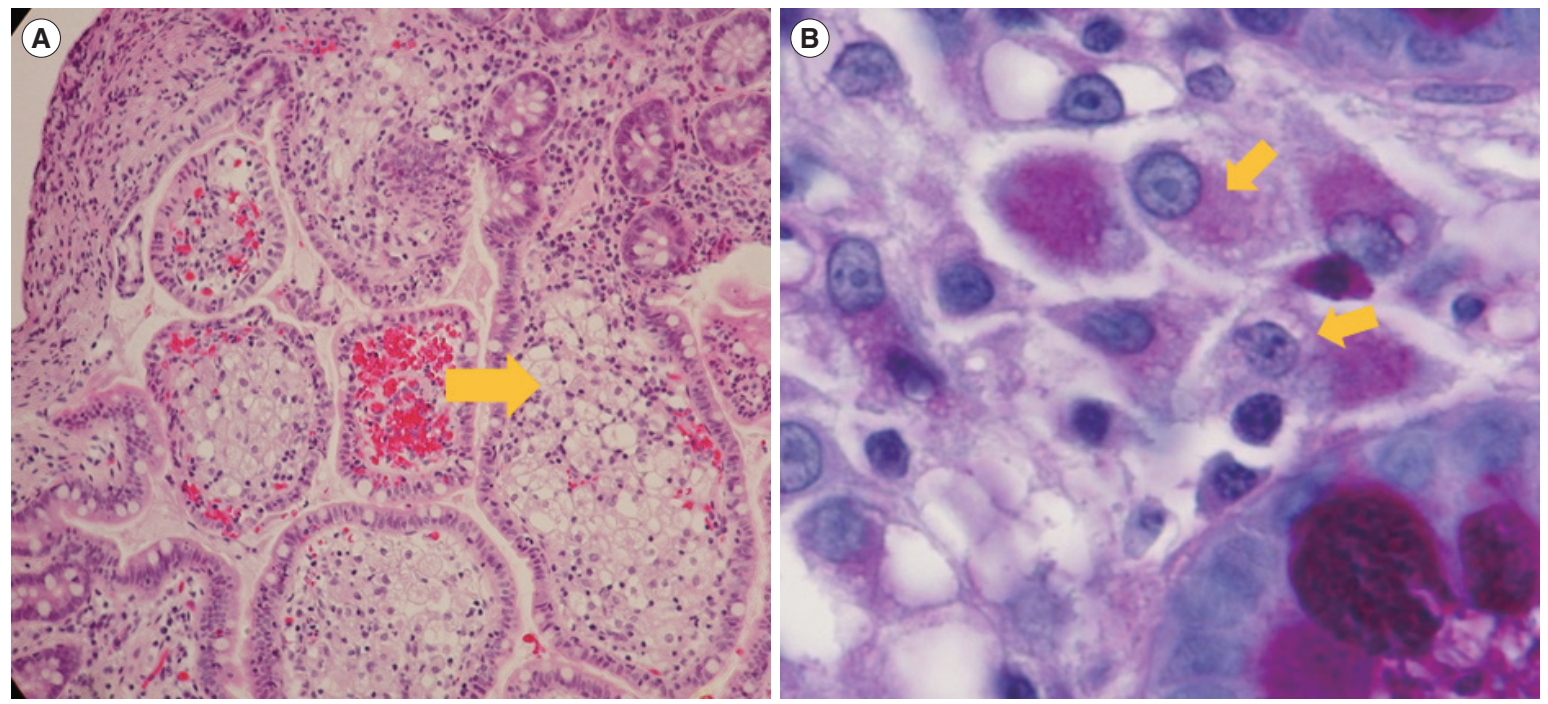

Fig. 2. Histological image of the biopsy specimens from the duodenum and terminal ileum revealed foamy macrophages with intracellular granules. Histological image of the terminal ileum with foamy macrophages. (A) Foamy macrophages are evident in the sample with H\&E staining (arrow $\times 100)$. (B) Periodic acid-Schiff positive macrophages are seen in the lamina propria (arrows, $\times 400$ ).

denoscopy. Small bowel capsule endoscopy showed mild erythema and edema throughout the small bowel. Colonoscopy revealed frank friability, marked erythema, absence of vascular patterns, and erosions throughout the rectum and sigmoid colon; these findings were similar to those of ulcerative colitis (Fig. 1). Her terminal ileum was also slightly edematous.

Mucosal biopsy specimens of duodenum and small colon showed increased inflammatory cells including neutrophils, plasma cells and lymphocytes in lamina propria. Crypt architectural distortions were also observed in the rectum and sigmoid colonic mucosa. Biopsy specimens from the duodenum and terminal ileum revealed foamy macrophages with intracellular granules (Fig. 2A). Periodic acid-Schiff staining and Ziehl-Neelsen staining were performed to exclude infectious disease caused by intracellular parasites such as Mycobacterium avium complex. The samples were positive for periodic acid-Schiff staining (Fig. 2B), but negative for Ziehl-Neelsen staining, suggesting that M. avium complex infection was unlikely. We suspected T. whipplei infection and performed electron microscopy, which showed rod-shaped bacillary bodies within a macrophage measuring $1 \mu \mathrm{m}$ in size (Fig. 3A). Furthermore, we performed polymerase chain reaction (PCR) 


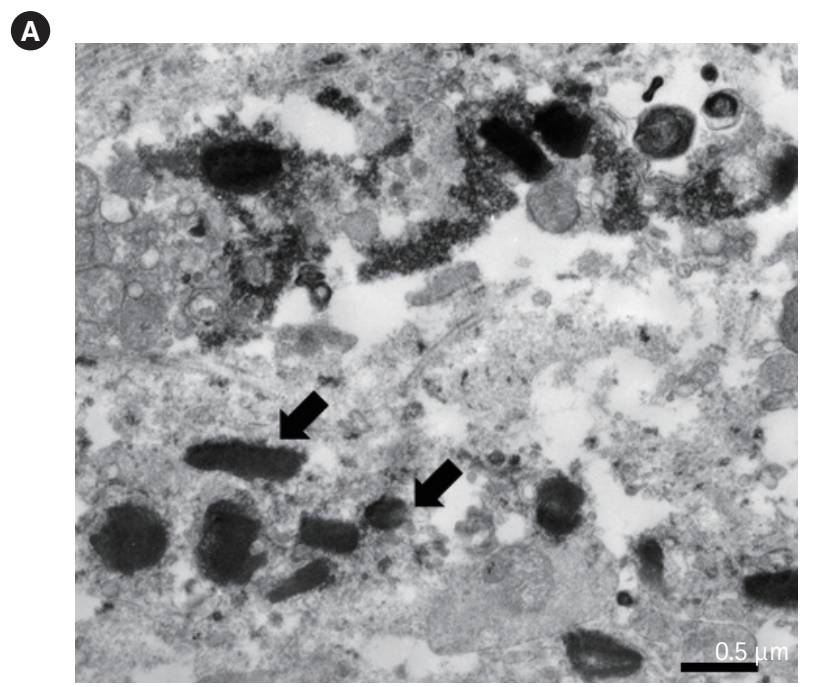

B

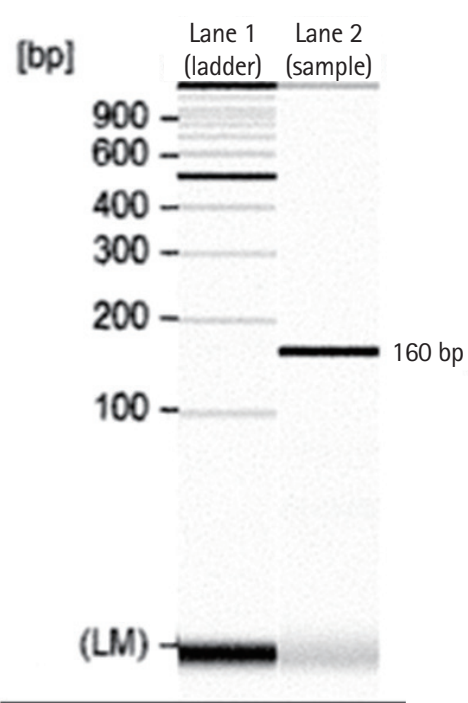

Fig. 3. Electron microscopic findings of the biopsy sample and polymerase chain reaction (PCR) detection of Tropheryma whipplei from the terminal ileum. (A) Numerous rod-shaped bacilli are observed (arrows). (B) The lane shows a PCR product of 160 base pairs specific to the T. whipplei $16 \mathrm{~S}$ ribosomal RNA.
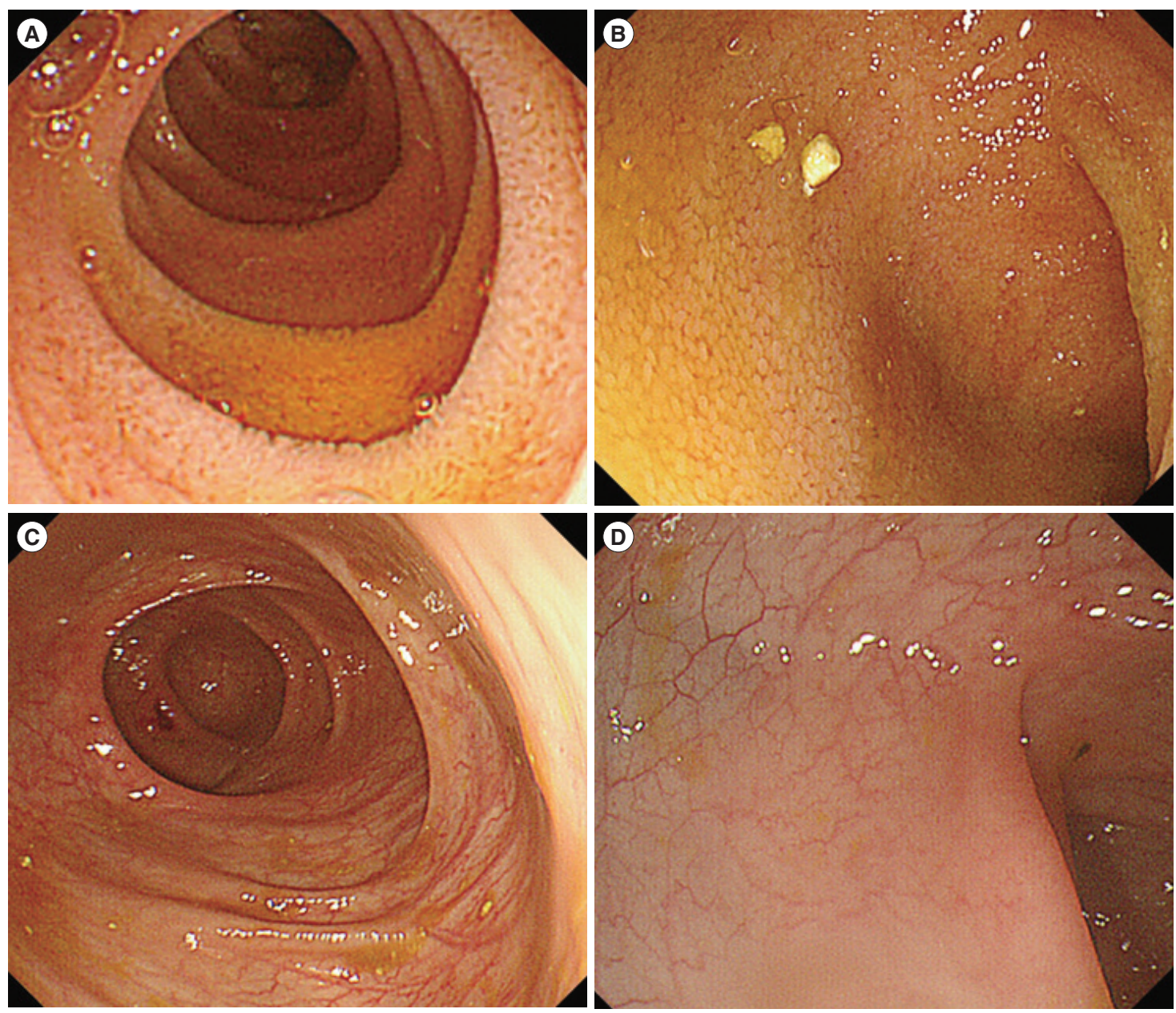

Fig. 4. Endoscopic findings after 12 months of treatment for Whipple disease. Duodenal edema is improved (A), as well as ileal (B) and colonic (C, D) pathological findings. 
using DNA from the duodenal mucosa and primers specific to whipplei DNA. ${ }^{6,7}$ A specific PCR product of 160 base pairs that corresponds to T. whipplei $16 \mathrm{~S}$ ribosomal RNA gene was detected (Fig. 3B). A definite diagnosis of WD was made. Laboratory tests for the evaluation of immunodeficiency, which frequently causes WD, including human immunodeficiency virus (HIV) antibody tests, CD4 T-cell count, lymphocyte-stimulation test, and natural killer T-cell activity test, were negative. We performed whole exome sequencing of DNA isolated from the blood cells of the patient and her parents to identify any underlying genetic diseases, including primary immunodeficiency diseases or monogenic IBD; no known specific mutations associated with these diseases were identified.

She was treated intravenously with ceftriaxone $(50 \mathrm{mg} / \mathrm{kg}$ per day) for 2 weeks, followed by oral therapy with sulfamethoxazole-trimethoprim (40 mg/kg per day). Diarrhea and hematochezia improved within a few days, and growth retardation recovered at 12 months after treatment initiation. Additional follow-up at 12 and 24 months showed improved mucosa edema in the small bowel and colon (Fig. 4). In the follow-up biopsy after 12 months of treatment initiation, $T$. whipplei was not detected by electron microscopy and the PCR did not amplify T. whipplei DNA specific amplicon. She continues taking oral therapy with sulfamethoxazole-trimethoprim without any symptoms.

\section{DISCUSSION}

The clinical symptoms and pathological findings of WD are diverse, and can involve the joints, central nervous system, heart, skin, lymph nodes, musculoskeletal system, eye, and small intestine. ${ }^{8} \mathrm{WD}$ is thought to be extremely rare. Its reported annual incidence is approximately 1 per 1,000,000 people and tends to affect middle-aged white men. ${ }^{9,10}$ Pediatric WD has mainly been associated with immunocompromise, especially following HIV infection in African countries. ${ }^{11}$ Only 2 pediatric cases of central nervous system infection without immunodeficiency have been reported. ${ }^{12,13}$ Fenollar et al. ${ }^{14}$ have reported that $T$. whipplei is associated with acute diarrhea based on data from 555 stool samples from children in France. However, their patients were negative for T. whipplei DNA after recovery from gastroenteritis. The researchers concluded that enteritis in those patients was different from WD, which has chronic symptoms. Günther et al. have proposed a diagnostic procedure for $\mathrm{WD}{ }^{9,15}$ To the best of our knowledge, the present case is the first of a pediatric patient with WD showing gastroenteritis diagnosed using this procedure.

The patient presented here did not seem to have any major acquired or primary immunodeficiency diseases. Whole exome sequencing was also performed; however, none of the candidate genes known to cause monogenic IBD or IBD susceptible genes were detected. This may indicate the importance of including T. whipplei infection in the differential diagnosis of chronic diarrhea in every patient, not only those with immunodeficiency.

Our patient was initially diagnosed with IBD. The fact that our patient had a family history of childhood-onset CD also supported the diagnosis of IBD. Pathological findings showed distortion of the crypt structure of the rectal and sigmoid colon mucosa, which is typical for IBD. However, the absence of basal plasmacytosis and crypt abscess in our patient were atypical findings for IBD. The fact that this case showed improvement in symptoms without any treatment for IBD also suggested that her intestinal inflammation was due to WD not IBD. Furthermore, histologic findings did not show any typical IBD findings during the follow-up period.

Chronic diarrhea is a recognized GI symptom of WD, whereas bloody diarrhea has not been described in WD. None of the guidelines or previous reports have suggested the need to exclude T. whipplei infection before making the diagnosis of IBD. However, improvement of GI symptoms after antibiotic treatment in the present case shows that bloody diarrhea can also be caused by WD. This might indicate that WD could present with IBD-like phenotypes and should be added to the differential diagnosis of IBD.

In our patient, first-line treatment with ceftriaxone for 2 weeks followed by oral trimethoprim-sulfamethoxazole for 1 year was effective. If the first-line therapy is not completely successful, penicillin G or ceftriaxone for 4 weeks followed by oral trimethoprim-sulfamethoxazole for 1 year is recommended as a second-line therapy. ${ }^{16}$

Marth $^{17}$ have published a review of 41 cases with WD after anti-tumor necrosis factor inhibitor (TNFI) treatment. The patients received TNFI for arthritis, which is thought to be a symptom of undiagnosed WD. Worsening WD after TNFI was reported in these cases, and symptoms included colitis, ulcer, and intestinal perforation. In addition, cases with sepsis, pericarditis, and a fatal case of immune-reconstitution inflammation syndrome due to WD were reported. Since the intestinal symptoms observed in patients with WD after TNFI therapy are similar to those often observed in patients with TNFI-resistant IBD, there may be some WD cases misdiagnosed as IBD 
and treated with TNFI. Since steroids, immunomodulators, and biologics, which are often used to treat IBD, may worsen WD symptoms, excluding this infectious disease is important. Furthermore, diagnosis of this infectious disease is essential since it can be treated with antibiotics. ${ }^{9}$

Although whole exome sequencing in our patient revealed no known variants associated with monogenic IBD, presence of unknown novel causative genetic variants cannot be ruled out. In addition, the sequencing results showed that she has a compound heterozygote variant in LRRK2 (c.T1532G, c.A713T). The gene is a known risk factor for developing Parkinson disease, CD, and Leprosy. This variant might be also associated with WD development, however, additional research including sequencing of the DNA of other WD patients is required for validation of our hypothesis linking the association of the LRRK2 with the development of the disease.

This is a novel case of WD in a child who developed symptoms resembling IBD, diagnosed based on the presence of $T$. whipplei DNA in the intestinal mucosa. Therefore, mucosal biopsy and PCR for T. whipplei DNA are important for the accurate diagnosis of $\mathrm{WD}$, even in children without immune dysfunction. These investigations should be performed in every patient before TNFI treatment.

\section{ADDITIONAL INFORMATION}

\section{Funding Source}

The authors received no financial support for the research, authorship, and/or publication of this article.

\section{Conflict of Interest}

No potential conflict of interest relevant to this article was reported.

\section{Author Contribution}

Conceptualization: Tatsuki M, Ishige T. Data curation: Tatsuki M, Ishige T, Igarashi Y, Hatori R. Methodology of functional studies: Hokama A, Hirato J, Muise A. Project administration: Takizawa T, Arakawa H. Writing - original draft: Tatsuki M, Ishige T. Writing- review and editing: all authors. Approval of final manuscript: all authors.

\section{Non-Author Contribution}

We thank Drs. Hiroshi Chinen and Atsushi Iraha in University of Ryukyus, for performing PCR for detecting Tropheryma whipplei DNA. We also thank Dr. Masanori Tanaka in Hirosaki
Municipal Hospital, and Dr. Ryuichi Wada in Hirosaki University for reviewing patient's histopathology.

\section{ORCID}

Tatsuki M

Ishige $\mathrm{T}$

Igarashi $\mathrm{Y}$

Hatori R

Hokama A

Hirato J

Muise A

Takizawa T

Arakawa $\mathrm{H}$

https://orcid.org/0000-0001-6430-9347 https://orcid.org/0000-0002-2409-7359 https://orcid.org/0000-0002-1828-3323 https://orcid.org/0000-0002-8110-3882 https://orcid.org/0000-0002-8310-9989 https://orcid.org/0000-0002-8278-6240 https://orcid.org/0000-0001-9624-3346 https://orcid.org/0000-0003-2169-0787 https://orcid.org/0000-0002-8014-2433

\section{REFERENCES}

1. Benchimol EI, Fortinsky KJ, Gozdyra P, Van den Heuvel M, Van Limbergen J, Griffiths AM. Epidemiology of pediatric inflammatory bowel disease: a systematic review of international trends. Inflamm Bowel Dis 2011;17:423-439.

2. IBD Working Group of the European Society for Paediatric Gastroenterology, Hepatology and Nutrition. Inflammatory bowel disease in children and adolescents: recommendations for diagnosis: the Porto criteria. J Pediatr Gastroenterol Nutr 2005;41:1-7.

3. Yardley JH, Hendrix TR. Combined electron and light microscopy in Whipple's disease: demonstration of "bacillary bodies" in the intestine. Bull Johns Hopkins Hosp 1961;109:80-98.

4. Yajima N, Wada R, Kimura S, et al. Whipple disease diagnosed with PCR using formalin-fixed paraffin-embedded specimens of the intestinal mucosa. Intern Med 2013;52:219-222.

5. Yogi T, Hokama A, Kinjo F, et al. Whipple's disease: the first Japanese case diagnosed by electron microscopy and polymerase chain reaction. Intern Med 2004;43:566-570.

6. Relman DA, Schmidt TM, MacDermott RP, Falkow S. Identification of the uncultured bacillus of Whipple's disease. N Engl J Med 1992;327:293-301.

7. Ramzan NN, Loftus E Jr, Burgart LJ, et al. Diagnosis and monitoring of Whipple disease by polymerase chain reaction. Ann Intern Med 1997;126:520-527.

8. Fenollar F, Puéchal X, Raoult D. Whipple's disease. N Engl J Med 2007;356:55-66.

9. Marth T, Moos V, Müller C, Biagi F, Schneider T. Tropheryma whipplei infection and Whipple's disease. Lancet Infect Dis 2016;16:e13-e22.

10. Fleming JL, Wiesner RH, Shorter RG. Whipple's disease: clini- 
cal, biochemical, and histopathologic features and assessment of treatment in 29 patients. Mayo Clin Proc 1988;63:539-551.

11. Patel SJ, Huard RC, Keller C, Foca M. Possible case of CNS Whipple's disease in an adolescent with AIDS. J Int Assoc Physicians AIDS Care (Chic) 2008;7:69-73.

12. Tan TQ, Vogel H, Tharp BR, Carrol CL, Kaplan SL. Presumed central nervous system Whipple's disease in a child: case report. Clin Infect Dis 1995;20:883-889.

13. Duprez TP, Grandin CB, Bonnier C, et al. Whipple disease confined to the central nervous system in childhood. AJNR Am J Neuroradiol 1996;17:1589-1591.

14. Fenollar F, Minodier P, Boutin A, et al. Tropheryma whipplei associated with diarrhoea in young children. Clin Microbiol Infect 2016;22:869-874.

15. Günther U, Moos V, Offenmüller G, et al. Gastrointestinal diagnosis of classical Whipple disease: clinical, endoscopic, and histopathologic features in 191 patients. Medicine (Baltimore) 2015;94:e714

16. Masselot F, Boulos A, Maurin M, Rolain JM, Raoult D. Molecular evaluation of antibiotic susceptibility: Tropheryma whipplei paradigm. Antimicrob Agents Chemother 2003;47:1658-1664.

17. Marth T. Systematic review: Whipple's disease (Tropheryma whipplei infection) and its unmasking by tumour necrosis factor inhibitors. Aliment Pharmacol Ther 2015;41:709-724. 